\title{
New Developments for Thermopile Array Sensors in Packages as small as TO39
}

\author{
M. Schnorr $^{1)}$, B. Forg ${ }^{1)}$, F. Herrmann ${ }^{1)}$, Dr. W. Leneke ${ }^{1)}$, Dr. J. Schieferdecker ${ }^{1)}$, M. Schulze ${ }^{1)}$, Dr. M. \\ Simon ${ }^{1)}$ K. Storck ${ }^{1)}$, L. Buydens ${ }^{2)}$ \\ 1): Heimann Sensor GmbH, Maria-Reiche-Str. 1, D-01109 Dresden, Tel. 0351-888885-0; \\ info@heimannsensor.com; \\ 2): Melexis Technologies, Transportstraat 1, B-3980 Tessenderlo
}

\begin{abstract}
Heimann Sensor further improved the sensitivity, the image quality and the distance dependency of the HTPA8x8. In addition Heimann Sensor announced the new HTPA16x4 with a fully digital $I^{2} \mathrm{C}$ connection, both fitting inside a TO39 housing.
\end{abstract}

Key words: Thermopile Array, TO39 Housing, HTPA8x8, improved IR sensitivity, infrared detection

\begin{abstract}
Introduction
Heimann Sensor has developed the first fully monolithic thermopile array and the world smallest thermopile sensor in TO housing [1].

Thermopile sensor arrays are a competitive solution against other technologies, wherever a thermal picture of the environment is needed and very high resolutions are not necessary, such as person detection, surveillance of temperature critical surfaces, hotspot detection, energy management and security cameras. Other applications can be found in industrial process control and air condition control [2].
\end{abstract}

The benefits of this technology are the low costs, the very small power consumption, the short time constant of the elements, as well as the high sensitivity of the system [3].

Now Heimann Sensor has further increased the quality of the sensors by changing the shape of the pixel and reducing the chip size.

\section{Improved sensitivity for the HTPA8x8}

The sensitivity of the HTPA8x8 was increased by a factor of 3.8 from $65 \mathrm{~V} / \mathrm{W}$ to $246 \mathrm{~V} / \mathrm{W}$. This was achieved via changing the shape of the pixel and filling the housing with inert gas for a reduced thermal conductivity.

Fig. 1 is showing a thermal picture with a person holding his hands up. The picture is taken and interpolated with the Heimann Array Software.

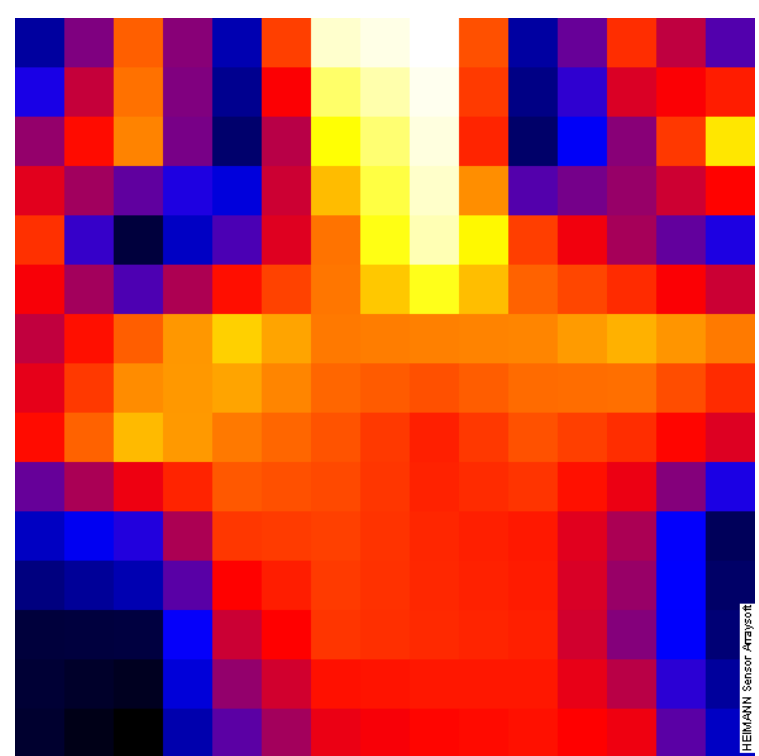

Fig. 1. Picture of the HTPA8x8 showing a person with hands up, taken and interpolated with the Heimann Sensor Arraysoft.

\section{Improved distance dependency for the HTPA8x8}

The distance dependency for the HTPA8x8 was measured with and without an aperture, which lead to significant improves of the distance dependency with the aperture.

In Fig. 2 a plot for the distance dependency for the HTPA8x8 with a L7.0 optics is shown. There the temperature difference to the temperature at an object distance of $14 \mathrm{~cm}$ is plotted versus the object distance. The object temperature was $40{ }^{\circ} \mathrm{C}$ and had a size of $38 \mathrm{x}$ $18 \mathrm{~cm}$. 


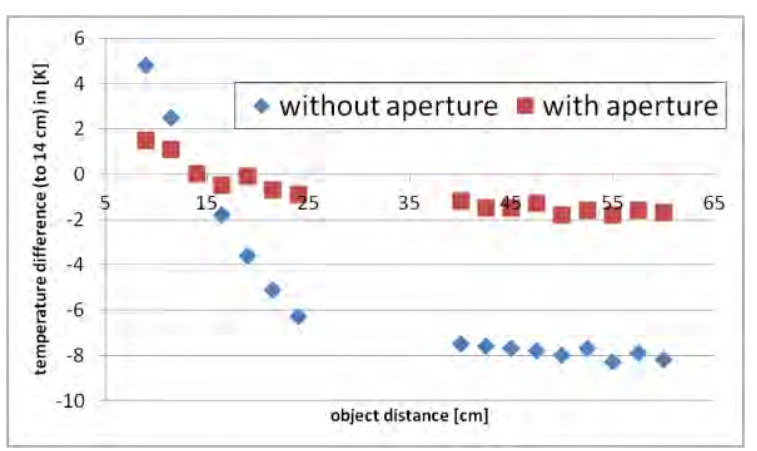

Fig. 2. Plot showing the distance dependency for the HTPA8x8 with a L7.0 optics

\section{Small chip size for the HTPA8x8}

Heimann Sensor developed a small HTPA8x8 chip to fit into the TO39 package with a diameter of only $9.1 \mathrm{~mm}$. A special TO39 header with 9 pins was chosen to fulfill the desired needs.

The TO39 housing with the HTPA8x8 on it can be seen in Fig. 3 . The pixels of the HTPA8x8 are shown in Fig. 4.

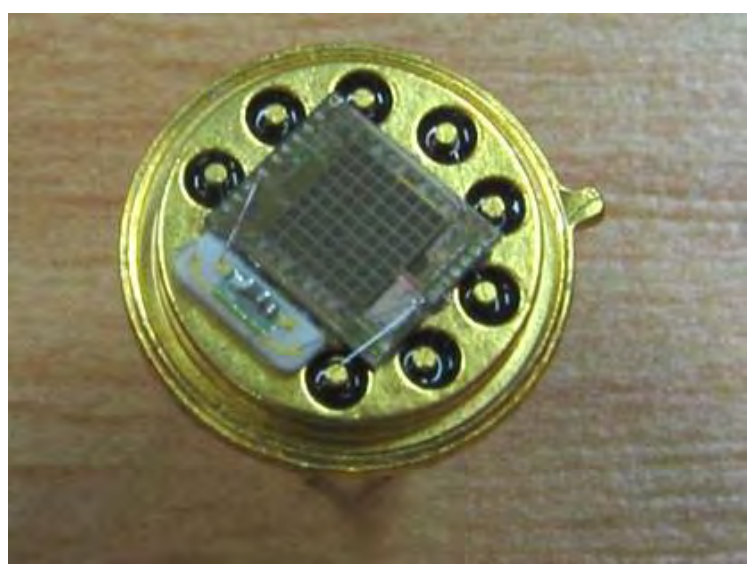

Fig. 3. Picture of the HTPA8x8 chip glued onto the TO39 header.

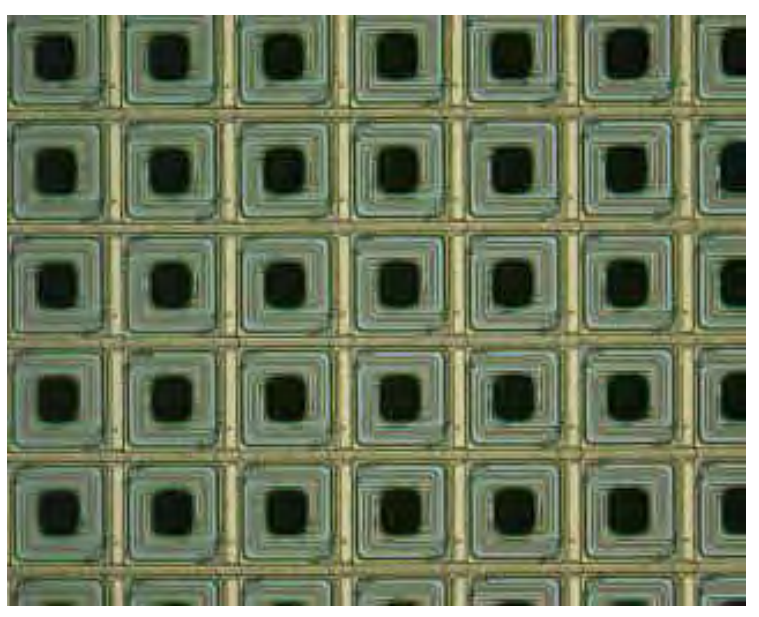

Fig. 4. Picture of the pixels of the HTPA8x8.

\section{HTPA16x4}

A new sensor with $16 \times 4$ thermopile elements was developed in cooperation with Melexis. This sensor has a built in EEPROM to store the calibration constants and a 16 bit ADC. Thanks to parallel data processing the refresh rate can be set up to $512 \mathrm{~Hz}$. Due to the small chip size and the $\mathrm{I}^{2} \mathrm{C}$ communication the sensor can be easily placed inside a four pin TO39 housing. The maximum temperature that can be measured is $800{ }^{\circ} \mathrm{C}$ and the Noise Equivalent Temperature Difference (NETD) can be as low as $0.07 \mathrm{~K}$ relative to $1 \mathrm{~Hz}$ refresh rate at an object temperature of $100{ }^{\circ} \mathrm{C}$.

The HTPA16x4 sealed with a L5.5 and open with its EEPROM glued onto the TO39 header can be seen in Fig. 5 .

A thermal image, taken and interpolated with the Heimann Array Software, showing a person wearing glasses is shown in Fig. 6 .

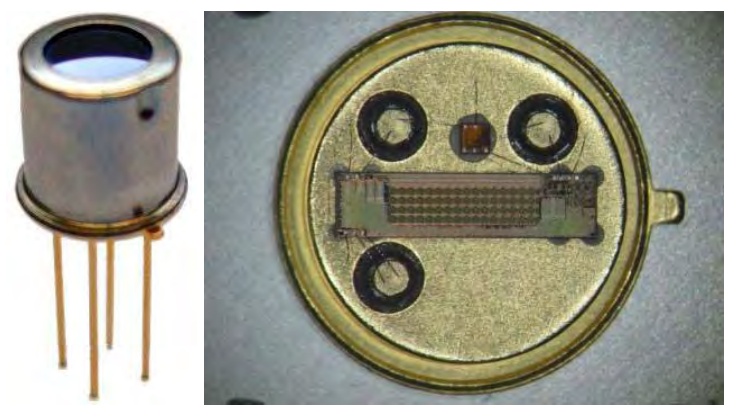

Fig. 5. Picture of the HTPA16x4 sealed and open in the TO39 housing. The EEPROM can be seen at the right picture on top.

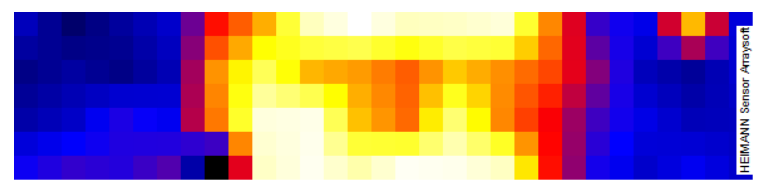

Fig. 6. Picture of the HTPA16x4 showing a person with glasses, taken and interpolated with the Heimann Sensor Arraysoft.

\section{NETD of the HTPA16x4 versus refresh rate}

The NETD for the HTPA16x4 was measured for different set refresh rates, the plot can be seen in Fig. 7. All data was calculated relative to a refresh rate of $1 \mathrm{~Hz}$.

Via a trimming register the achieved frame rate can be fine tuned. With this a more detailed measurement was done for different set refresh rates, the frame rate is then a combination of the refresh rate and the trimming register. The plot is shown in Fig. 8. The object temperature was $25^{\circ} \mathrm{C}$ for both measurements. 


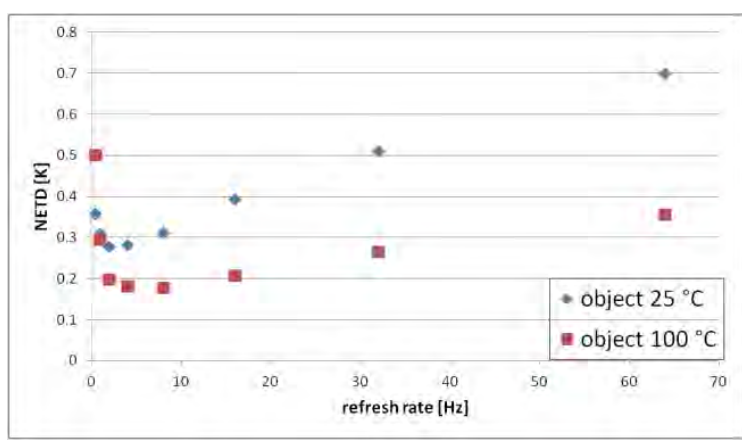

Fig. 7. Plot of the NETD versus the refresh rate for the HTPA16x4 for an object temperature of 25 and $100^{\circ} \mathrm{C}$.

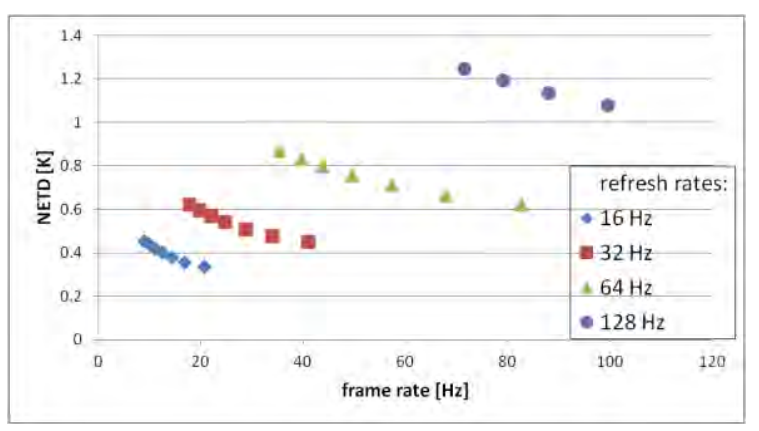

Fig. 8. Plot of the NETD versus the frame rate for the HTPA16x4 for an object temperature of $25{ }^{\circ} \mathrm{C}$ and with fine tuning of the frame rate via the trimming register.

\section{Different optics}

We tested several optics with different focal lengths for the TO39 housing. In Tab. 1 the corresponding fields of view (FOV) in degree are listed. The quadratic HTPA8x8 has the same FOV in both directions, the HTPA16 $\times 4$ has a rectangular field of view. The first given value is valid for the long dimension (16 pixel) and the second one for the short dimension (4 pixel).

Because of the same housing all lenses are interchangeable.

Tab. 1: Calculated FOV in ${ }^{\circ}$ for different TO39 optics for different sensor types

\begin{tabular}{|c|c|c|c|c|}
\hline $\begin{array}{c}\text { Lens } \\
\text { (focal } \\
\text { length) }\end{array}$ & L2.85 & L3.6 & L5.5 & L7.0 \\
\hline $\begin{array}{c}\text { HTPA } \\
8 \times 8\end{array}$ & 46 & 37 & 25 & 20 \\
\hline $\begin{array}{c}\text { HTPA } \\
16 \times 4\end{array}$ & $63 \times 18$ & $52 \times 14$ & $36 \times 9$ & $28 \times 7$ \\
\hline
\end{tabular}

\section{Noise Equivalent Temperature Difference (NETD)}

The measured NETD in Kelvin for several optics is listed in Tab. 2. Here all optics were made of uncoated silicon for a better comparison, the empty cells can be estimated to be similar to the measured values of other optics for the same array type. The ambient temperature was always set to $25{ }^{\circ} \mathrm{C}$ and the object temperature to either $25{ }^{\circ} \mathrm{C}$ (first value) or $100{ }^{\circ} \mathrm{C}$ (second value). The refresh rate was $42.2 \mathrm{~Hz}$ for the HTPA8x8 and $16 \mathrm{~Hz}$ for the HTPA16x4. The NETD was then calculated relative to $1 \mathrm{~Hz}$.

Tab. 2: NETD in $K$ for different TO39 optics for different sensor types; first values are for $25^{\circ} \mathrm{C}$ and second for $100^{\circ} \mathrm{C}$ object temperature

\begin{tabular}{|c|c|c|c|c|}
\hline $\begin{array}{c}\text { Lens } \\
\text { (focal } \\
\text { length) }\end{array}$ & L2.85 & L3.6 & L5.5 & L7.0 \\
\hline $\begin{array}{c}\text { HTPA } \\
8 \times 8\end{array}$ & - & - & - & 0.20 \\
\hline HTPA & - & 0.19 & 0.16 & - \\
$16 \times 4$ & & 10.10 & 10.07 & \\
\hline
\end{tabular}

\section{Maximum Object Temperatures}

The maximum object temperatures that can be measured are listed in Tab. 3 with the different lens types. The ambient temperature is assumed to be $25{ }^{\circ} \mathrm{C}$, lower ambient temperatures will lead to lower possible object temperatures. In order to increase the maximum object temperature, filters and apertures can be added. Doing so will lead to less resolution but a higher possible object temperature range.

The stated values for the HTPA8x8 are for the new redesigned chip where the amplification is a factor 3 lower. The values marked with $a$ * are only estimated values.

Tab. 3: Maximum measureable object temperatures in ${ }^{\circ} \mathrm{C}$ for different TO39 optics for different sensor types

\begin{tabular}{|c|c|c|c|c|}
\hline $\begin{array}{c}\text { Lens } \\
\text { (focal } \\
\text { length) }\end{array}$ & L2.85 & L3.6 & L5.5 & L7.0 \\
\hline $\begin{array}{c}\text { HTPA } \\
8 \times 8\end{array}$ & $350^{*}$ & 350 & 350 & 300 \\
\hline $\begin{array}{c}\text { HTPA } \\
16 \times 4\end{array}$ & $800^{*}$ & 800 & $800^{*}$ & $750^{*}$ \\
\hline
\end{tabular}




\section{High speed sensors}

Heimann Sensor has put a lot of effort into the speed of the sensors. The HTPA8x8 achieves a refresh rate of up to $120 \mathrm{~Hz}$ and the HTPA16x4 up to $512 \mathrm{~Hz}$.

\section{Different module types and Array Software}

We have developed and tested samples with different electronic interfaces such as SPI, UDP, UART and CAN. UART, SPI and CAN interfaces are perfect for system integration. For an easy start an Array Software was developed aswell, so that the UDP module is simply plug-and-play via a LAN cable. The screenshots of the thermal images (Fig. 1 and Fig. 6) have been taken with this software. We have built for our tests a prototype of a camera, a so called application set, this can be seen in Fig. 9 .

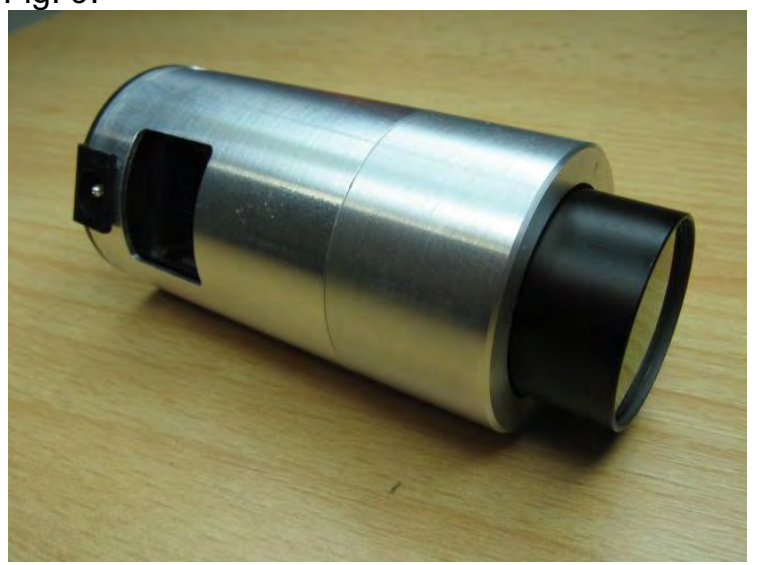

Fig. 9. Application Set with a UDP interface for an easy start and plug-and-play.

\section{References}

[1] W. Leneke, J. Schieferdecker, M. Schulze, M. Simon, K. Storck, B. Forg, F. Völklein: "Thermopile Linear Array Sensors and Modules with Short Time Constant and High Sensitivity"; Sensor Conference 2007, Proc. Vol.1, Nürnberg, Germany, p. 329-333

[2] B. Forg, W. Leneke, J. Schieferdecker, M. Schulze, M. Simon, K. Storck: "Thermopile Sensor Arrays with Internal Amplifiers and Digital Out"; Sensor \& Test 2008, Proceedings, Nürnberg, Germany, p. 249-252.

[3] B. Forg, F. Herrmann, W. Leneke, J. Schieferdecker, M. Schulze, M. Simon, K. Storck, F. Völklein: "New generation of high resolution and low cost Thermopile Arrays for Infrared Imaging"; Sensor \& Test 2010, Proceedings, Nürnberg, Germany, Section A5. 